Revista científica, arbitrada e indizada, bajo la modalidad electrónica.

Recibido: 23/10/2019

Aceptado: 04/11/2019

\title{
Perfil del contador público desde su ámbito de formación profesional
}

Profile of the public accountant from your professional training area

Cira Fernández de Pelekais

Florida Global University

cpelekais@gmail.com

cira.fernandez@floridaglobal.university

Elmar Aldrin Pelekais

Universidad Autónoma del Caribe

SUMMA University

pelekais@gmail.com

Carlos Farfán

SUMMA University

carlos.farfan@summauniversity.us

\section{RESUMEN}

Con el propósito de analizar el perfil del contador público desde su ámbito de formación profesional, se realiza un estudio en Colombia, Ecuador, México, Perú y Venezuela. Bajo un enfoque postpositivista cualitativo, catalogado por su naturaleza de tipo documental, utilizando como apoyo central el procedimiento comparativo. Debido a la cantidad de material bibliográfico consultado, el método analítico deductivo sirve de estrategia a los fines de efectuar el análisis de las categorías seleccionadas. Adicionalmente, se sustenta en un diseño interpretativo-crítico y bibliográfico. Queda claro del análisis efectuado, que en los cinco países examinados, el diseño curricular de esta carrera, esta soportado por consistentes bases para la construcción, además generación del conocimiento, asociado a la experiencia, objetividad, independencia y actuar ético, lo cual incide de manera exitosa en el desempeño laboral de este tipo de profesional.

Palabras Clave: Contador Público; Formación profesional, Diseño curricular. 


\title{
Revista científica, arbitrada e indizada, bajo la modalidad electrónica.
}

\begin{abstract}
With the purpose of analyzing the profile of the public accountant from his professional training field, a study is carried out in Colombia, Ecuador, Mexico, Peru and Venezuela. Under a qualitative postpositive approach, cataloged by its documentary nature, using the comparative procedure as central support. Due to the amount of bibliographic material consulted, the deductive analytical method serves as a strategy to carry out the analysis of the selected categories. Additionally, it is based on an interpretive-critical and bibliographic design. It is clear from the analysis carried out that in the five countries examined; the curricular design of this degree is supported by consistent bases for the construction, in addition to the generation of knowledge, associated with experience, objectivity, independence and ethical action, which affects successful way in the work performance of this type of professional.
\end{abstract}

Key words: Public Accountant; Professional training, Curriculum design.

\section{INTRODUCCIÓN}

Considerada una de las carreras más versátiles a nivel mundial, por la pertinencia de la actividad a cumplir, así como los contenidos a gestionar, los cuales resultan útiles en cualquier contexto geográfico, independientemente de su ubicación, en términos generales el diseño curricular en las Instituciones de Educación Superior no guarda grandes diferencias, probablemente algunas universidades son más innovadoras que otras.

No obstante, las competencias generales y específicas que este tipo de instituciones refuerza en la formación impartida en sus aulas, son propias del desempeño en el ejercicio de la profesión.

Con respecto a este planteamiento, para Roncancio et al., (2017), la carrera contable no queda fuera de la discusión sobre competencias, debido a los procesos de internacionalización y estandarización no solo de las normas de presentación de información financiera, sino de los procesos implícitos en la formación planificada para disciplinas como la contabilidad. Para los citados autores, estas posturas son generadas dentro de un ambiente algo más institucional, estrechamente atado al progreso y desarrollo de la profesión, en especial en la vertiente anglosajona, donde se ha propendido por la unificación de la manera como se prepara la información de carácter financiero (antes 


\section{Revista científica, arbitrada e indizada, bajo la modalidad electrónica.}

información contable) a partir del proceso de dispositivos de carácter técnico, conocidos como estándares internacionales de contabilidad en la actualidad.

En cuanto al estado del arte, han sido revisados algunos trabajos en los cuales fueron abordadas las mismas categorías analizadas en este artículo, tales como: Elizalde (2019), quien desarrolló una publicación sobre el "Perfil profesional del contador público". Para este autor, es posible evidenciar algunas coincidencias en la formación recibida en las diferentes instituciones universitarias en las que han cursado estudios. Entre ellas, destacan las competencias tanto generales como específicas, por ser una carrera que conlleva un trabajo independiente y responsable, sustentado en una sólida formación ética.

También Morales Alvarado (2016), hace su aporte al señalar que en el Perú las diferentes universidades en las cuales la carrera de Contabilidad no ponen énfasis en la calidad de la enseñanza centrado en el estudiante, donde el docente cumple un papel fundamental para el logro del aprendizaje, el Estado ha tomado acciones a través de regulaciones a fin de garantizar las cuestiones básicas de calidad. De igual forma, los docentes para el desarrollo de las materias incorporadas en el plan de estudios, deben responder al perfil del egresado definido por la institución educativa, y si este no contempla aspectos acorde a los cambios globales, el desempeño del profesional contable no será idóneo.

Por su parte, Mantilla et al., (2018), refieren que las instituciones de educación superior, tienen el compromiso de formar a los futuros profesionales con habilidades, destrezas y competencias adecuadas que le permitan desenvolverse con eficiencia, eficacia e idoneidad en el desempeño profesional. Por ello, este estudio estuvo enfocado en determinar la relación existente entre el componente ético, las capacidades y las competencias propias de la formación del contador-auditor. Arrojando como evidencia explica una alta correlación entre las competencias académicas, las capacidades y los valores recibidos en el aula como preparación para afrontar el campo laboral.

Por otro lado, este artículo fue trabajado bajo un enfoque postpositivista cualitativo, catalogado por su naturaleza de tipo documental, utilizando como apoyo central el procedimiento comparativo. Debido a la cantidad de material bibliográfico consultado, el método analítico deductivo ha servido de estrategia a los fines de efectuar el análisis de las 


\section{Revista científica, arbitrada e indizada, bajo la modalidad electrónica.}

categorías seleccionadas. Adicionalmente, se sustenta en un diseño interpretativo-crítico y bibliográfico.

Partiendo de las premisas anteriormente expuestas, se socializa un trabajo con el propósito de analizar el perfil del contador público desde su ámbito de formación profesional.

\section{MARCO TEÓRICO REFERENCIAL}

\section{Rol del contador público}

Para Moreno et al., (2012), citado por Morales (2016), el contador público en su rol debe ser:

Una persona asertiva, creativa, dotada de cualidades humanísticas con aptitud y actitud para el proceso de cambio preparado para las exigencias y requerimientos que el país exige, con pensamiento analítico, metódico, riguroso, compromiso ético en su formación y desarrollo profesional (p.21).

Por su parte, Montenegro (2017), afirma que en la actualidad, el contador público, está involucrado con la ética, moral y la profesión, ya que, todo lo expresado se encuentra inmerso en los principios orientadores del Código de Ética del Contador, en otras palabras, son lineamientos éticos para la formación profesional. De igual forma, este Código contribuye en impulsar al contador para tomar su trabajo con responsabilidad.

\section{La formación contable en las aulas}

A los fines de examinar esta temática, se abordan los aportes de Aguilar et al., (2018), para quienes la intervención de la formación contable en las aulas, se ha venido desarrollando por medio de modelos que incluyen una gran diversidad de estrategias instruccionales, con el objetivo de adecuarlos a distintos propósitos.

Por otro lado, según Montilla (2016), señala en la enseñanza de la contabilidad el uso del aprendizaje por descubrimiento, en donde el estudiante, en vez de recibir los contenidos de forma pasiva, descubre en los conceptos sus relaciones, los reordena para adaptarlos a su esquema cognitivo. De allí, la utilidad del Aprendizaje asistido, en el cual el docente/facilitador, desarrolla actividades de autogestión, ambas complementadas por un 


\section{Revista científica, arbitrada e indizada, bajo la modalidad electrónica.}

conjunto de ayudas que le permitirán al estudiante sentirse acompañado durante todo su proceso de aprendizaje.

\section{El Contador Público desde diferentes contextos}

Con el propósito de examinar los elementos contenidos en este artículo, son develados los aportes referenciales explorados, a la luz de la realidad geográfica analizada, en los siguientes términos:

\section{Realidad en Colombia}

Se inicia el abordaje teórico con la revisión de Ceballos y Revelo (2017), quienes en el artículo publicado, realizan la siguiente exposición: Frente a los problemas de corrupción que existen en la mayoría de las organizaciones empresariales de los países latinoamericanos, particularmente en Colombia, es necesario analizar desde la apreciación de egresados y empleadores, la propuesta formativa de la academia, así como los requerimientos de formación que el mercado laboral necesita del contador público.

Considerando, que el ejercicio de la contabilidad se gestiona por normas éticas que contemplan una serie de principios que suponen una garantía del comportamiento moral de este tipo de profesional. Sin embargo, se continúan presentando faltas morales en el ejercicio de los contadores, tal como se evidencia en la realidad internacional. Asimismo, enfatizan en que el contador público está en la obligación, tanto social, como profesional, de ser un abanderado de la verdad, promulgar siempre la ética, el respeto a la profesión, promover dentro de su gremio la transparencia, además de honestidad en cada acto de su ejercicio.

Según Díaz Ortega (2017), para explorar el ideal de formación integral del contador público, tomando como contexto las universidades públicas y privadas de la ciudad de Cúcuta en Colombia, es importante señalar que los docentes poseen un concepto de formación integral conformado por varias dimensiones, el cual contribuye al desarrollo social. En consecuencia, para lograr la participación del contador público como agente de cambio, transformador de la realidad a partir de los conocimientos recibidos, se necesita 


\section{Revista científica, arbitrada e indizada, bajo la modalidad electrónica.}

una integración entre la teoría y la práctica, con el fin que el contador tenga la competencia suficiente para solventar problemas de su entorno.

Aquí puede apreciarse de acuerdo a los resultados expuestos por la investigadora, el afianzamiento del perfil profesional debe estar fundamentado en la ética, pero adicionalmente es necesario integrar los contenidos programáticos con sus vivencias en el campo de trabajo, es decir, articular los conocimientos teóricos con el quehacer práctico.

Para Ríos León (2013), la cualificación académica de los estudiantes, es potenciada por la investigación la cual permite crear y desarrollar competencias, a los fines de ir fortaleciendo, además observando en el desarrollo de su carrera, permitiéndoles vigorizar su formación integral.

En consecuencia, según el autor, el rendimiento académico se ve reforzado en la medida en que el estudiante comprende cómo se desarrolla el programa que está cursando, como se va articulando con su proyecto de vida y como se proyecta su futuro como profesional de la Contaduría Pública.

También Roncancio et al., (2017), hacen su aporte al señalar: la dirección que ha tomado en las últimas décadas la formación en contabilidad, si bien mantiene los rezagos de la educación tradicional, ha sido direccionada a mantener la premisa de formar para el trabajo, en donde prevalece el saber hacer por encima de otro tipo de estructura de enseñanza aprendizaje. Asimismo, dentro de la formación en contabilidad existen una serie de problemáticas, pero una de estas es el "desarrollo de habilidades", las cuales son de interés inmediato. En la actualidad, frente a los cambios evidenciados a causa de la globalización de la economía y los avances tecnológicos, emerge con gran fuerza el discurso de competencias en la Educación Superior y por ende en los programas de formación superior en Colombia, los cuales refuerzan exigencias educativas, articulando ese aprendizaje al entorno laboral.

Con respecto a lo mencionado con anterioridad, Ariza et al., (2016), aseguran que existe una débil articulación en la formación profesional del contador público por ciclo propedéutico, en este caso, la integración de saberes no se maneja de forma sistémica, quedando vacíos por las diferencias en las competencias y perfiles de cada tipo de 


\section{Revista científica, arbitrada e indizada, bajo la modalidad electrónica.}

formación, adicional a ello la autonomía institucional que posibilita organizar de manera individual los sistemas educativos amplia la brecha diferencial frente a la formación tradicional, no se realiza de manera uniforme, dificultando la libre escogencia vocacional o movilidad.

\section{Realidad en Ecuador}

La aplicación del juicio profesional que debe realizar el contador en el desempeño de sus actividades, de acuerdo con Panchi (2017), es importante porque debe definirse como por ejemplo, el uso correcto de las estimaciones y provisiones, lo cual es esencial ya que incide directamente en los resultados de la información financiera.

Según el citado autor, el juicio profesional permite a los contadores combinar la teoría con la práctica contable, partiendo del desarrollo de habilidades críticas que admiten aplicar conocimientos técnicos adquiridos y la experticia de forma adecuada en el desempeño de las labores relacionadas con la contabilidad, asimismo, la auditoria e incorporar el conocimiento trasmitido de otros contadores para ampliar la base de conocimientos, a su vez el entendimiento profundo de normas contables, principios y valores para la elaboración del proceso contable.

De igual forma, Arellano et al., (2017), en el estudio sobre pertinencia y la determinación de la demanda insatisfecha de la carrera de Contabilidad y Auditoría, evidencian que existen herramientas para la construcción de un nuevo currículo que se ajusta tanto a los requerimientos como necesidades de la sociedad, promoviendo la integración de los saberes ancestrales, al igual la interculturalidad, con formación humana y ética, capaz de responder al mundo laboral. Según los referidos autores, el rediseño curricular de la carrera responde a los requerimientos empresariales, además a las nuevas competencias requeridas por el estado, administración tributaria, empresas públicas y privadas, para esto se determinaron en el objeto de estudio, los objetivos de formación de la carrera, el perfil profesional, quedando demostrado que los elementos están alineados a las nuevas tendencias nacionales e internacionales.

Agregando, que la construcción de la misión, visión, objetivos, perfil de egreso, perfil profesional del contador, competencias generales, se articulan a los objetivos del Plan 


\section{Revista científica, arbitrada e indizada, bajo la modalidad electrónica.}

Nacional del Buen Vivir, la problemática socioeconómica, los requerimientos laborales. De lo expuesto se evidencia, que la carrera de contabilidad y auditoría, incorpora nuevas competencias a la sociedad del conocimiento como desafío para superar las barreras intelectuales creadas en la formación ocupacional, acordes a los señalamientos del Plan Nacional del Buen Vivir 2013-2017, la Agenda para la Transformación Productiva 2010 2013, la propuesta de cambios en el conocimiento de la Matriz Productiva, Agenda Zonal y Provincial 2011; Plan de Desarrollo Chimborazo Minga por la Vida 2015 - 2020, las corrientes, además requerimientos de los actores.

\section{Realidad en México}

De acuerdo a lo planteado por Vargas et al., (2015), en México como en otros países ha aumentado la necesidad de que toda organización desempeñe su rol social, en este sentido, las Instituciones de Educación Superior (IES) no son ajenas a éste fenómeno, requiriendo la transformación de su quehacer académico refrendando su compromiso con la sociedad a través de una gestión universitaria, al igual política educativa que involucre y beneficie a su entorno, redimensionando sus perfiles, filosofía institucional y sobre todo la formación de profesionales líderes del cambio social, quienes se convierten en pilares del desarrollo sostenible con visión social, humanista, además ética.

Considerando, que la sociedad está viviendo momentos de transición urgiendo establecer sinergias entre los actores garantes del desarrollo colectivo: Gobierno, Empresas, Organizaciones Sociales, y fundamentalmente las Instituciones de Educación Superior, las cuales deben responder, además asegurar el cumplimiento de sus demandas.

En relación a las universidades, es necesario distinguir su gran capacidad de autotransformación en función de las exigencias sociales, económicas, tecnológicas, ambientales, se requiere diseñar un nuevo perfil del egresado universitario en contaduría, cuyo objetivo central sea la formación de los profesionales con un enfoque social, a lo que se ha denominado en la actualidad como Responsabilidad Social, que le aseguren un lugar en el mercado laboral. 


\section{Revista científica, arbitrada e indizada, bajo la modalidad electrónica.}

Complementa lo expuesto Mendoza et al., (2016), para quienes el área de Contabilidad ha permanecido por muchos años dentro de la oferta educativa de estudios superiores en México, primero egresando Contadores Públicos, después Licenciados en Contaduría. Ante las necesidades del mercado laboral actual, el Contador Público se ve en la penuria imperiosa de reinventarse, continuar siendo una pieza clave en el ámbito de la contabilidad, las finanzas y la administración. Es decir, en el ámbito de los negocios. Por ello, el Contador Público debe poseer una actitud de servicio hacia la sociedad, habilidades cognoscitivas y socio profesionales, sirviendo de apoyo directo o indirecto a la alta dirección, orientando su visión, de lo operativo a lo estratégico, que en su función de evaluadores se conviertan en críticos de estados financieros nacionales e internacionales.

La presencia de la contabilidad en el mundo de los negocios actual se convalida siempre y cuando ofrezca un servicio de calidad a sus clientes La contabilidad tiene como objetivo generar información útil para la toma de decisiones de usuarios tales como administradores, banqueros, inversionistas, dueños, público en general, empleados, autoridades gubernamentales y otros más.

\section{Realidad en Perú}

Para Guerra Campos (2018), en la contabilidad, el desarrollo del prototipo del uso para la toma de decisiones y las explicaciones teóricas conocidas como "investigación", parten de la presencia de los usuarios de la indagación y la multitud de múltiples intereses implementados. En consecuencia, pensar en las carencias de los interesados en cuanto a la información que pidan para la toma de decisiones, no es tan solo de gran importancia, por otra parte, se establece una base para un adecuado progreso de la teoría, fundamentando la constitución de procedimientos contables.

El contador público es un informador del proceso económico en los mercados a nivel nacional e internacional, con la seguridad que sus conocimientos ayudan a la elaboración de los informes, utilizando un rol consciente, ejecutivo y participativo. Frente a esta idea, es pertinente la siguiente pregunta, ¿Cuál es el nivel de relación del rol del contador público y la contabilidad social?. Pues el contador público debe tener la facultad para demostrar las 


\section{Revista científica, arbitrada e indizada, bajo la modalidad electrónica.}

evidencias económicas; pero no solo eso, sino también resulta necesario saber todos los sucesos que se desenvuelven en el entorno empresarial y social.

De acuerdo a este planteamiento, los estudiantes de contaduría, a partir del inicio de sus vidas académicas, vislumbran que las instituciones les trazan metas dirigidas a tolerar en el mercado, sin embargo, para la carrera contable toma un gran valor cuando produce un modelo de estandarización que empieza a juntar todas las operaciones e interacciones de una organización. En este sentido, el contador público tiene conocimientos financieros para tomar decisiones, guiando las acciones de las empresas, la idea no es buscar solamente el beneficio, sino también el compromiso con la sociedad. Después de todos los progresos y contribuciones a la contabilidad en la actualidad, se ha creado un nuevo modelo para tener en cuenta los factores influyentes en la sociedad como lo es el tema ambiental, en otras palabras, se enfrenta distintos desafíos; desistir del lado cuantitativo, porque en muchos términos la contabilidad es concebida de manera financiera, por medio de una visión mecanicista reduciéndose a tan solo aspectos legales.

Por su parte, Dextre Flores (2013), destaca la importancia de la formación humanística del estudiante de la carrera de contabilidad teniendo en cuenta el carácter social del conocimiento contable, cuyos principales objetivos son la preparación, interpretación y evaluación de la información financiera de los eventos económicos producidos. Mencionando que el plan de estudios adoptado en algunas instituciones de Perú, predispone al egresado de la disciplina contable, a elaborar juicios morales positivos y a entender la educación profesional como una parte de la formación humana.

Finalmente, Tua Pereda (2012), señala que la Contabilidad, representa una dimensión desbordante, al insertarse en un marco de trascendencia vital, convirtiéndose en un instrumento básico de optimización económica, social y, en consecuencia, favorecer, potenciando el Desarrollo económico, al perfeccionamiento del género humano. Como el mismo autor lo asegura, no hay duda de que esta dimensión está presente en los orígenes de la disciplina, tanto en el florecer comercial del Mediterráneo como en el quipu andino, ámbitos en los cuales, indudablemente, ha contribuido a racionalizar los respectivos sistemas económicos; pero no es menos cierto que es en las modernas economías 


\section{Revista científica, arbitrada e indizada, bajo la modalidad electrónica.}

desarrolladas o en vías de crecimiento, donde aquella trascendental misión de la Contabilidad aparece con perfiles más nítidos.

\section{Realidad en Venezuela}

Según Zamora (2015), la Contaduría Pública, en los procesos de formación impartidos en los centros académicos, ha llevado a que el reconocimiento como profesión se dé a través de la regulación local. Por ello, los procesos formativos en un gran número de Programas de Contaduría Pública en el país, basan sus estructuras curriculares "plan de estudios", en la mera regulación contable, bien local o Internacional (NIC, NIIF), olvidando los desarrollos teóricos de la contabilidad, y el cómo se argumenta el ser de esta, vista desde los objetos; así, entonces, se deja entrever que la "formación profesional” se sostiene, en el contexto del hacer.

En este orden de ideas, Grisanti (2014), realiza una investigación sobre la labor ejercida por los contadores públicos, señalando tal como ocurre con otras profesiones liberales, tiene un marco de responsabilidades que deben cumplirse para que su trabajo sea llevado adelante de manera satisfactoria. Los contadores públicos por excelencia ejercen su profesión de manera independiente, esto es, los auditores externos, quiénes tienen como actividad principal emitir un dictamen para dar fe pública sobre estados financieros de las empresas contratantes. En consecuencia, un contador público, ya sea por negligencia o complicidad, emita una opinión diferente a lo indicado por las circunstancias, puede causar serios perjuicios a los terceros, lo cual le podría generar graves consecuencias de muy diversa naturaleza.

Refiere el citado autor, que el ejercicio profesional del contador público está regido directa e indirectamente por muchas leyes en el marco del ordenamiento jurídico de cualquier país, y por ende sanciones por su incumplimiento, las cuales pueden ir desde el resarcimiento de daños y perjuicios como consecuencia de su inadecuada actuación profesional, hasta llegar inclusive en otros casos de mayor gravedad, a medidas privativas de libertad del contador público, ello en ocasión de cometer delitos que ameriten la aplicación de penas de esta naturaleza. 
Por último, Montaner y Perozo (2008), en una investigación ejecutada con respecto a la formación ética del contador público en la Universidad del Zulia en Venezuela, evidenciaron como los estudiantes manejan conocimientos vagos en cuanto a los términos de ética, moral y valores; asimismo, desconocen los valores que deben practicar según lo planteado en el plan curricular y en el código de ética de su carrera. Concluyendo que, la institución no ha sido efectiva en cuanto a la formación ética de sus estudiantes, por lo cual deben tomarse acciones correctivas e implementar un sistema transversal de educación en valores.

\section{MATERIALES Y MÉTODO}

Este trabajo está fundamentado en un enfoque postpositivista cualitativo, catalogada por su naturaleza de tipo documental, utilizando como apoyo central el método comparativo para poder analizar el perfil del contador público desde su ámbito de formación profesional.

Por otro lado, debido a la cantidad de material bibliográfico consultado, el método analítico deductivo sirve de estrategia a los fines de efectuar el análisis de las categorías seleccionadas. Adicionalmente, se sustenta en un diseño interpretativo-crítico y bibliográfico.

Con estos fines se acudió a conformar el universo de material objeto de estudio, quedando constituido por revistas y otras publicaciones periódicas, apoyándose en buscadores en línea, revistas científicas y libros. Entre las fuentes de datos utilizadas, destacan las bases Scopus, Dialnet, SciELO, Redalyc y Saber-ULA, trabajos de grado, entre otros. Luego se procedió a definir las unidades básicas de donde recolectar la información analizada, codificándolas en correspondencia con las categorías conceptuales. (Pelekais et al., 2015).

En relación a lo planteado, Arias (2016), señala que la investigación documental es un proceso basado en la búsqueda, recuperación, análisis, crítica e interpretación de datos secundarios, es decir, los obtenidos y registrados por otros investigadores en fuentes documentales: impresas, audiovisuales o electrónicas. 


\section{RESULTADOS}

Queda claro del análisis efectuado, que en los cinco países examinados, el diseño curricular de esta carrera, esta soportado por consistentes bases para la construcción de una propuesta innovadora en materia contable, además generadora de conocimiento asociado a la experiencia, objetividad, independencia, actuar ético, lo cual incide de manera exitosa en el desempeño laboral de este tipo de profesional. Asimismo, los hallazgos develados dejan evidente, que el perfil del contador público, no está atado a las condiciones geográficas de cada país, sino por lo que representa la profesión, fundamentada en la credibilidad y actuación ética, centrada en principios socialmente responsables. De igual forma, se pudo corroborar, la necesidad de actualización, sobre todo en materia de instrumentos legales, además de normativas internacionales, esenciales para la sostenibilidad en tiempo y espacio.

\section{CONCLUSIONES}

Con base en los resultados derivados de la revisión del arqueo bibliográfico examinado, se concluye que quienes egresan como Contador Público, en cualquier Institución de Educación Superior, obtienen una formación acorde a las exigencias y necesidades del mercado laboral, donde deben insertarse, articulando las competencias requeridas. Sin embargo, es necesario que desde estudiantes busquen insertarse en un trabajo acorde a su formación para adquirir experiencia.

Además es recomendable que estudien la posibilidad de utilizar simuladores contables, para aprender haciendo y desarrollar o fortalecer competencias profesionales. De igual forma, el docente en este campo de estudios, debe promover el desarrollo de los procesos cognitivos de los estudiantes, guiando la intervención y cuidando las características individuales, haciendo uso de las estrategias instruccionales.

En función de lo planteado, es importante tener claro el requerimiento de formar un Contador Público, que además de cubrir las exigencias académicas de la carrera, aprobando todos los contenidos programáticos contenidos en el respectivo diseño curricular, también 


\section{Revista científica, arbitrada e indizada, bajo la modalidad electrónica.}

tenga competencias digitales, formación basada en valores y el principio de responsabilidad de sus acciones.

Se necesita un Contador Público capaz de transformar la realidad empresarial desde su ámbito de acción profesional, porque de ese actuar responsable, va a depender la efectiva toma de decisiones en una organización.

\section{REFERENCIAS BIBLIOGRÁFICAS}

Aguilar Anaya, M; Saavedra García, M; Tapia Sánchez, B. (2018). "Las estrategias instruccionales en la enseñanza y la formación del Contador". Perspectivas, Año 21 - No 41 - mayo. pp. 93-116. Universidad Católica Boliviana "San Pablo", Unidad Académica Regional Cochabamba.

Arellano Cepeda, O; Coronel, J; Cáceres, L; Zavala, J (2017). Estudio de la demanda insatisfecha para el rediseño curricular de la Carrera de Contabilidad y Auditoría, Universidad Nacional de Chimborazo en el Ecuador. INNOVA Research Journal 2017, Vol 2, No. 8: 53-68.

Arias, F. (2016). El proyecto de investigación. Introducción a la metodología científica $7^{\circ}$ Edición. Caracas Venezuela: Editorial Episteme.

Ariza Angarita, Y; Villasmil Molero, M; Monsalve Peláez, M; Fontalvo Anaya, F (2016). Análisis crítico de la formación del contador público por ciclos propedéuticos en la globalidad compleja. Universidad Libre Seccional Barranquilla-Colombia/ Universidad Simón Bolívar-Colombia. Revista Desarrollo Gerencial, 8 (2) Pp. 144 160.

Ceballos Gómez, H; Revelo Córdoba, L (2017). La formación ética del contador público, un requerimiento del contexto social y laboral colombiano. Revista Perspectiva Empresarial ISSN: 2389-8194 (En línea), [S.1.], v. 4, n. 2, p. 13-25, sep. ISSN 2389-8194 (En lí. Disponible en: <https://rpe.ceipa.edu.co/index.php/perspectivaempresarial/article/view/128/60>. Fecha de acceso: 5 enero. 2020 doi:http://dx.doi.org/10.16967/rpe.v4n2a2.

Dextre Flores, J. (2013). Los retos de la formación por competencias del contador público. Contabilidad y Negocios (8) 16, 2013, pp. 35-47 / ISSN 1992-1896

Díaz Ortega, N. (2017). Formación integral del contador público: Una aproximación desde el punto de vista de los docentes. Revista ESPACIOS. ISSN 07981015 Vol. 38 (N 50) Año 2017.

Elizalde, L. (2019). Perfil profesional del Contador Público. Revista Digital Publisher. Año 4 / No. 5-1:208-216. Quito (Ecuador) /Edición especial - Septiembre/ ISSN 2588-0705. 


\section{Revista científica, arbitrada e indizada, bajo la modalidad electrónica.}

Grisanti, A. (2014). Responsabilidad del contador público en el ejercicio independiente de su profesión. Revista Actualidad Contable. FACES. 17 (29), 18-48. Maracaibo. Venezuela.

Guerra Campos, D. (2018). Rol del Contador Público y Contabilidad Social en las empresas mineras del distrito de San Borja, Año 2017. Trabajo de Grado Universidad César Vallejo. Perú. Disponible en: http://repositorio.ucv.edu.pe/bitstream/handle/UCV/23393/Guerra_CDR.pdf?sequen $\underline{\text { ce }=1 \& \text { is Allowed }=y}$

Mantilla, M; Tovar, G; Arias, M; Ríos, G. (2018). Competencias del contador-auditor en el perfil de egreso. Caso Universidad Técnica de Ambato, Ecuador. Actualidad Contable FACES ISSN E.: 2244-8772. Año 21 No 37, Julio - Diciembre. Mérida. Venezuela (90-117).

Mendoza, L; Ramírez, J; Cuarenta, S; Santos, O; Mares, J. (2016). Análisis Comparativo entre el perfil de egreso del Contador Público y las exigencias del mercado laboral. Revista Iberoamericana de Ciencias. Vol. 3. No.7. ReIbCi - Diciembre www.reibci.org. Disponible en: http://www.reibci.org/publicados/2016/dic/2000777.pdf

Montaner, J; Perozo, J. (2008). Formación ética del Contador Público: Caso: Facultad de Ciencias Económicas y Sociales de la Universidad del Zulia. Revista de Ciencias Sociales, 14(2), 379-387. Recuperado en 11 de septiembre de 2018, de http://www.scielo.org.ve/scielo.php?script=sci_arttext\&pid=S131595182008000200013\&lng=es\&tlng=es.

Montenegro, G. (2017). La responsabilidad social del contador público: diseño de un código de ética. Título de Ingeniera en Contabilidad y Auditoría CPA. Universidad Técnica de Machala - Ecuador. Recuperado de: http://repositorio.utmachala.edu.ec/bitstream/48000/10756/4/ECUACE-2017CADE00539.pdf

Montilla, A. (2016). Consideraciones sobre las estrategias de enseñanza más efectivas en la contabilidad. Negotium, Vol. 12, No. 34, pp. 23- 57.

Morales Alvarado, L. (2016). Factores influyentes en la formación profesional contable en el Perú en un marco globalizado. Trabajo de Grado Universidad de Buenos Aires. Disponible en: http://bibliotecadigital.econ.uba.ar/download/tpos/15020793_MoralesAlvaradoL.pdf.

Panchi, V. (2017). El contador en ejercicio de su profesión y el juicio profesional. Revista Órbita Pedagógica. Publicação quadrimestral. Vol. 4, Año 2017, No. 3 (SetembroDezembro). Pp 1-13.

Pelekais, C; El Kadi, O; Seijo, C; Neuman, N (2015). El ABC de la investigación. Guía didáctica. Venezuela: Ediciones Astro Data S.A. 
Ríos Urrutia, G. (2017) “Los principios y valores como base para la formación de competencias profesionales del estudiante de noveno semestre de la Carrera de Contabilidad y Auditoría de la Universidad Técnica de Ambato.” Tesis Universidad Técnica de Ambato. Ecuador

Roncancio García, Á; Mira Alvarado, G; Muñoz Murcia, N. (2017). Las competencias en la formación del profesional contable: una revisión de las posturas institucionales y educativas en Colombia. Revista Facultad de Ciencias Económicas, [S.1.], v. 25, n. 2, p. 83-103, sep. 2017. ISSN 1909-7719. Disponible en: https://revistas.unimilitar.edu.co/index.php/rfce/article/view/3070/2605>. Fecha de acceso: 10 sep. 2018 doi:https://doi.org/10.18359/rfce.3070.

Tua Pereda, J. (2012). Contabilidad y desarrollo económico. Revista Contabilidad y Negocios, vol. 7, núm. 13, 2012, pp. 94-110. Departamento Académico de Ciencias Administrativas Lima, Perú.

Vargas, R; Cruz, E; Gatica, L; García, P. (2015). El Contador Público como líder del cambio social en el nuevo escenario educativo mexicano. Revista Iberoamericana de Ciencias. Vol. 2 No.4: 166-177. México.

Zamorano, E. (2017). Desarrollo ético de los profesionales de la contaduría y la administración. Instituto mexicano de contadores 